\title{
Modifying a thermoplastic polyurethane for improving the bonding performance in an adhesive technical process
}

\author{
Geovana de Avila Bockorny ${ }^{1,3}$, Maria Madalena Camargo Forte ${ }^{1}$, Stephani Stamboroski ${ }^{2,3}$, Michael Noeske ${ }^{3}$, \\ Andreas Keil ${ }^{3}$ and Welchy Leite Cavalcanti $i^{3^{*}}$
}

\section{${ }^{*}$ Correspondence:}

welchy.leite.cavalcanti@ifam. fraunhofer.de

${ }^{3}$ Fraunhofer Institute

for Manufacturing

Technology and Advanced

Materials IFAM, Wiener Straße

12, 28359 Bremen, Germany

Full list of author information is available at the end of the article

\begin{abstract}
The present work describes pretreatment processes of thermoplastic polyurethane elastomer (TPU) material, used as an adhesive in powder form, that results in the increased peeling resistance of joints. The TPU in powder form is an environmentallyfriendly adhesive and provides safer bonding conditions in the shoe manufacturing industry. TPU particles are soft and require an anti-blocking agent to prevent agglomeration. However, particles covered by such an anti-blocking agent may account for a low peeling resistance in the resulting joints. To overcome this disadvantage, two pretreatment processes for the TPU adhesive were evaluated. The results of a two-step wet washing process, representing a conventional solvent-based procedure that involves both the application of a solvent and a powder separation step, are compared to the performance of a one-step dry plasma treatment process. The process type did not affect the size and agglomeration behavior of the particles, and both processes were effective in improving joint strength according to peeling test results. Spectroscopic investigation of the particle surface after washing or plasma treatment indicated some zinc concentration, which is interpreted to result from the anti-blocking agent. The crystallinity of the TPU, investigated by thermal analysis, was higher after pretreatment. The obtained results indicate that TPU in powder form is a versatile adhesive for the shoe industry with a safer application compared to solvent based adhesive systems.
\end{abstract}

Keywords: TPU adhesive, Powder adhesive, Zinc stearate, Atmospheric pressure plasma, Surface analysis, Shoe industry

\section{Background}

Thermoplastic polyurethane (TPU) is an interesting class of polymers which have versatile applications, mainly as thermoplastic adhesives. TPUs are linear block copolymers obtained by polymerization of three basic components, a diisocyanate and a short (chain extensor) and long-chain diol [1]. TPUs have alternating flexible and rigid segments connected by covalent links, as the diagram shows in Fig. 1. The soft segment, a high molecular weight macrodiol polyester, has a low polarity, while the rigid crystallizable segment, a diisocyanate linked to a low molecular weight diol (short-chain diol) or diamine, has a high density of urethane groups and a more pronounced polarity. At

\section{Springer Open}

(0) 2016 de Avila Bockorny et al. This article is distributed under the terms of the Creative Commons Attribution 4.0 International License (http://creativecommons.org/licenses/by/4.0/), which permits unrestricted use, distribution, and reproduction in any medium, provided you give appropriate credit to the original author(s) and the source, provide a link to the Creative Commons license, and indicate if changes were made. 


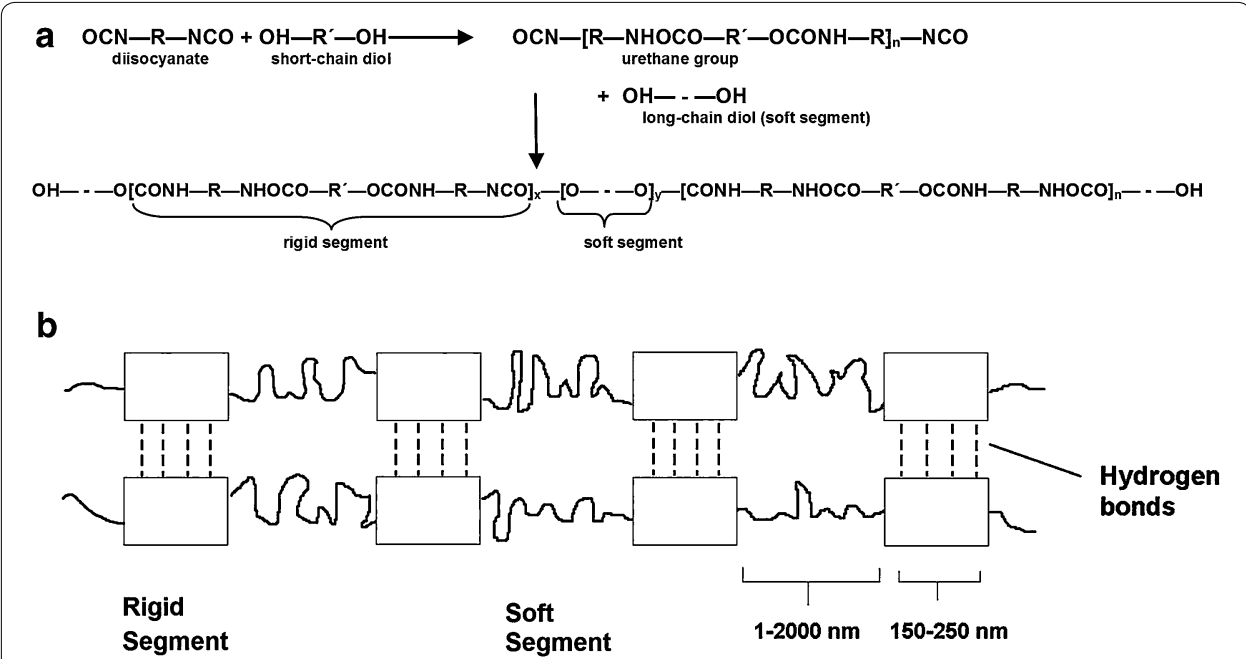

Fig. 1 Thermoplastic polyurethane chemical reaction (a) and block structure configuration (b)

room temperature, the TPU morphology comprises dispersed hard crystalline domains in a soft matrix of flexible segments [2].

There is a peculiar type of polyurethanes in which the hard segment content is very low and the long-chain diol is very crystalline and, therefore, the crystalline fraction is formed by the soft segments and a lower concentration of hard segments. This type of TPU presents adhesive properties above the melting point and is used as a high performance adhesive in the shoe industry [3].

TPU adhesives are usually applied to substrates in the form of a solution, and the removal of the organic solvent is a labour-intensive process [2]. TPU can also be used, in powder form, as a hot melt adhesive, whereby the TPU powder is applied in a thin layer to the substrate. It is then melted by heat treatment, wetting the substrate, and upon subsequent cooling it develops bonding properties [4].

To obtain the TPU adhesive in powder form it is necessary to submit the polymer material to a milling process. This procedure is delicate and has an impact on the TPU properties when used as adhesive. The process of powder adhesive production is not a simple one, and requires anti-blocking additives that are normally introduced during the milling process, and which are also of benefit to the storage of the product. The mechanics of the grinding process for a peripheral adhesive have recently been investigated [5], and it was found that during this process temperatures above the glass transition temperature of the adhesive can be reached locally. Such a temperature influence during the processes may cause undesired effects in the grinding result.

A satisfactory adhesion during an adhesive bonding process is achieved when there is a wetting, or even an infiltration, of the melted adhesive in the substrate surface, as well as a good cohesive force due to the formation of crystallites during the hardening of the adhesive [6]. An increase in the amorphous fraction of the TPU is usually sufficient to ensure good adhesion properties. However, this is not suitable for the milling process [1]. A flexible amorphous phase favors particle agglomeration in the milling equipment, thus anti-blocking agents and lubrication need to be used [7]. An additive that is widely 
used in the industry is zinc stearate, a hydrophobic dispersant, which interacts with the adhesive particles and prevents agglomeration and blocking during the milling, storage, and application processes. A previous study [8] elucidates that the presence of zinc stearate in powder TPU adhesives limits their bonding performance. Zinc stearate effects an inadequate bonding between the substrates, as revealed by a low peel resistance compared to a TPU adhesive with a reduced quantity of zinc stearate. The milling process using zinc stearate is shown in Fig. 2.

Zinc stearate is an amorphous solid that is insoluble in water and polar solvents such as alcohol and ether. It is a powerful release agent and lubricant, much used in the rubber industry [9]. The action of this organic salt as a lubricant occurs due to its thermal insulation properties, avoiding heat transfer [10,11].

For an investigation into an approach to reduce the lubricating action of zinc stearate or stearic acid in the adhesive layer during the bonding procedure, two different technologies were considered and a process optimization was performed for each. The challenge was to develop a fast and selective treatment process of the milled TPU powder, i.e., partially removing the parting agent without compromising the particle size distribution of the TPU material. The two techniques are as follows:

\section{Washing process}

A conventional and manual process, in which the polymer is washed with an organic solvent, such as the hydrocarbon mixture white spirit, after the milling process. The solvent dissolves zinc stearate (and stearic acid) without dissolving the TPU.

\section{Plasma process}

The plasma in an atmospheric pressure plasma (APP) process comprises chemically active species and a wide variety of components, such as excited and ionized molecular and atomic species, neutral radicals and energetic photons. The reactive environment in contact with the TPU particle surface leads to chemical reactions that generate active sites, polar groups and products resulting from the cleavage and subsequent modification of the bonds at the surface [12].

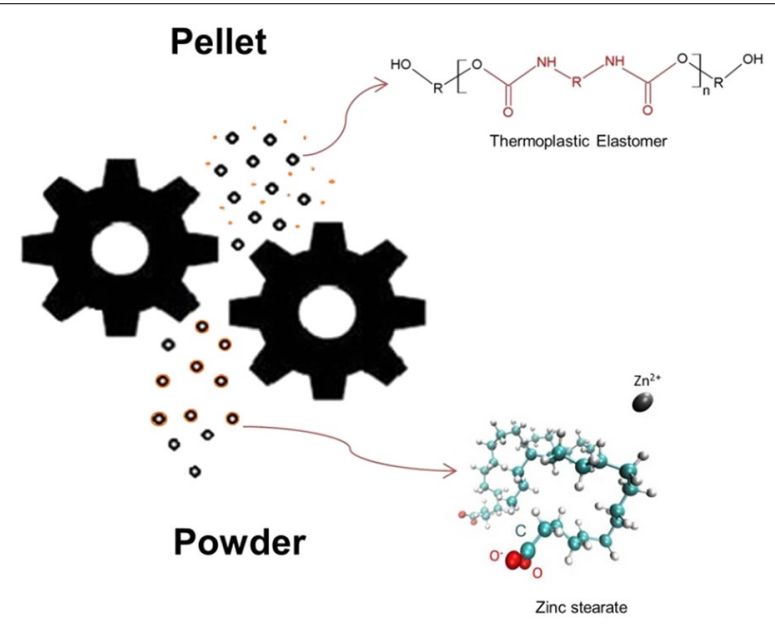

Fig. 2 Diagram showing the basics of the milling process with zinc stearate 


\section{Methods}

The thermoplastic elastomer, polyester-based polyurethane (TPU), with a molecular weight of $10,000 \mathrm{~g} / \mathrm{mol}$, a viscosity of $1800 \mathrm{cPs}$ [15\% methyl ethyl ketone (MEK)], a glass transition temperature of $-42{ }^{\circ} \mathrm{C}$, was used in the pellet form and constitutes a previously described material [13]. Zinc stearate $\left[\mathrm{Zn}\left(\mathrm{C}_{17} \mathrm{H}_{35} \mathrm{COO}\right)_{2}\right]$ with an ash content of $12.8-13.8 \%$, a free fatty acid content of less than $1.5 \%$, a melting point between 120 and $122{ }^{\circ} \mathrm{C}$, and a particle size of less than 200 mesh $(74 \mu \mathrm{m})$ was purchased from the Baerlocher company. The organic solvent white spirit with around $17 \%$ of aromatics was purchased from Sigma Aldrich (terpenaline, no 86460-2.5L Lot BCBQ3376V). The organic salt and solvent were used as received from the manufacturers.

\section{Experimental procedure}

The APP process that was developed at the Fraunhofer Institute IFAM was carried out using a Reinhausen atmospheric pressure plasma nozzle. Figure 3 shows a simplified schematic diagram of the plasma process, in which part of the zinc stearate can be oxidized and polymer molecules on the surface layer of the TPU can be modified. A removal of the zinc from the particles due to the action of the plasma is fairly improbable; however, a mechanical impact within the vortex might influence the material composition. A wall temperature of up to $120^{\circ} \mathrm{C}$ may be expected and a heat transfer to the polymer powder may be avoided, resulting in the temperature being sufficiently low to avoid the melting of the TPU adhesive during the plasma treatment.

The experimental data obtained in bonding tests were analyzed by single factor ANOVA variance type, followed by post hoc Fisher's LSD type, with a significance level of $5 \%$ [14]. This is a commonly used statistical test and aims to determine whether there is a significant difference between the mean values for each test and whether the independent factors influence the dependent variables [15].

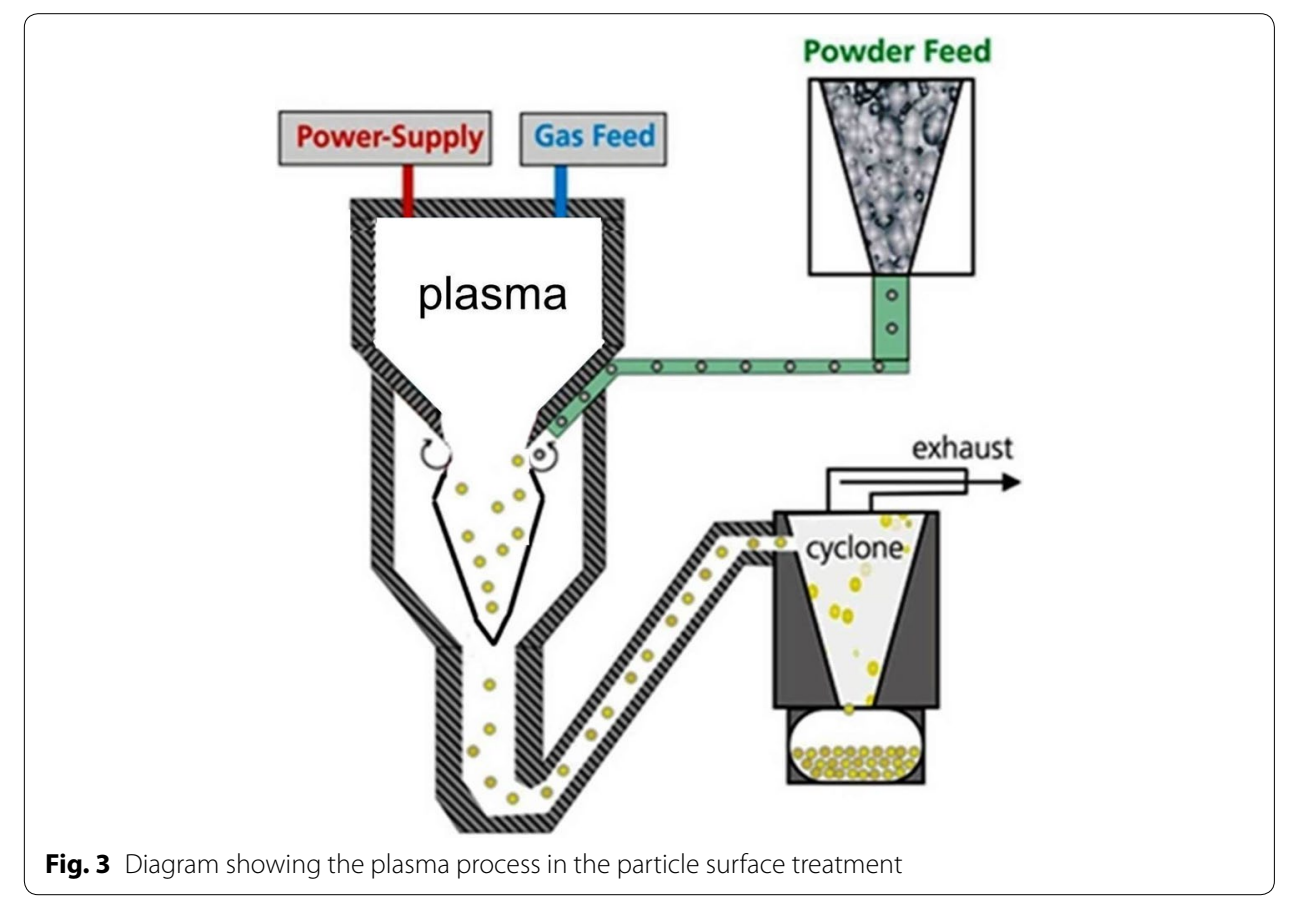


Optical microscopy analysis

A morphological investigation of the TPU particles was carried out using a digital microscope, VHX 500 from Keyence and a Keyence VHZ 100 lens, with a magnification factor of 100 .

\section{Beckman Coulter investigation}

The size distribution of TPU particles was evaluated using Beckman Coulter equipment, with ethanol as an immersion liquid. This investigation was performed at ambient temperature.

\section{SEM analysis and EDX investigations}

The SEM examination of the samples was performed in a Field Emission Scanning Electron Microscope (FESEM), type FEI Helios 600 (Dual Beam). The resolution is $0.9 \mathrm{~nm}$ at $15 \mathrm{kV}$ at optimal working distance and $1 \mathrm{~nm}$ at $15 \mathrm{kV}$ at the coincidence point. The images of the sample surface were generated at acceleration voltages between 0.35 and $30 \mathrm{kV}$ at working distances between 1 and $10 \mathrm{~mm}$. For the detection of secondary or backscattered primary electrons an Everhart-Thornley or an in-lens detector was used, and for the STEM studies (scanning transmission electron microscopy) a bright field, dark field and 12-segment HAADF detector (high-angle annular dark field) was used. For cryo-SEM investigations, a Quorum PP2000T preparation-system was available. Energy Dispersive X-ray analysis (EDX) measurements were performed with an Oxford $\mathrm{X}$-Max80 silicon drift detector (SDD) with an ATW2-window and an energy resolution down to $129 \mathrm{eV}$, the detection angle of the detector was $35^{\circ}$.

\section{XPS investigations}

Investigations of the surface composition were performed with X-ray Photoelectron Spectroscopy (XPS) applied to small piles of the powder samples. XPS spectra with an information depth of around $0.01 \mu \mathrm{m}$ were taken using a Kratos Ultra system applying excitation of photoelectrons by monochromatic $\mathrm{Al} \mathrm{K}_{\alpha}$ radiation within an area of approximately $0.2 \mathrm{~mm}^{2}$. The system was operated at a base pressure of $4 \times 10^{-8} \mathrm{~Pa}$, the sample neutralization was performed with low energy electrons $(<5 \mathrm{eV})$. An electrostatic lens was used, the take-off angle of electrons was $0^{\circ}$, and the pass energy was fixed to $20 \mathrm{eV}$ (or, respectively, $40 \mathrm{eV}$ in case of some less concentrated constituents) in high resolution spectra and $160 \mathrm{eV}$ in survey spectra. Elemental ratios were calculated based on the area of the peaks and considering relative sensitivity factors.

\section{DSC analysis}

The thermal analyses of the TPU samples $(5.5 \mathrm{mg})$ were carried out in a differential scanning calorimetry (DSC) Q100 equipment from TA Instruments (according ASTM D3418-12_1 [16]), in a T Zero non-hermetic aluminum pan, at a heating rate of $10{ }^{\circ} \mathrm{C} /$ min. A nitrogen flow of $50 \mathrm{~mL} / \mathrm{min}$ was maintained in the DSC oven. The samples were heated to $80{ }^{\circ} \mathrm{C}$, annealed for $5 \mathrm{~min}$, then cooled to $-34^{\circ} \mathrm{C}$. The TPU crystallinity content $\left(\mathrm{X}_{\mathrm{c}}\right)$ was determined using the equation: $\mathrm{X}_{\mathrm{c}}=\left(\Delta \mathrm{H}_{\mathrm{m}} / \Delta \mathrm{H}_{0}\right) \times 100$, where $\Delta \mathrm{H}_{\mathrm{m}}$ is the measured polymer melting heat and $\Delta \mathrm{H}_{0}$ is the melting heat of the crystalline polymer [17], which amounted to $196.8 \mathrm{~J} / \mathrm{g}$ [18]. 


\section{Peel resistance}

The peel test was carried out according to ABNT NBR 10456:2012 (Adhesives for Footwear and Resistance Bonding [19]). The substrates used were injected specimens of polyvinyl chloride (PVC), with a hardness of $75( \pm 5)$ Shore A, a width of $30( \pm 1) \mathrm{mm}$, a length of $160( \pm 2) \mathrm{mm}$ and a thickness of $3( \pm 1) \mathrm{mm}$. The procedural steps for the joint preparation using the PVC specimens and for the adhesive evaluation are shown in Table 1.

\section{Results and discussion}

The TPU adhesive was investigated with a focus on the particle size and surface composition of the powder samples both before and after submitting the powder to either the washing process with white spirit or the plasma treatment. The effect of these treatments on the characteristics of the powder is discussed regarding the thermal behavior of the TPU samples and the bonding properties of the PVC joints. The discussion suggests a structural model for the particles.

\section{Evaluation of powder particle size and surface composition}

The properties of the powdered raw materials entering the milling process were determined after which the powdered TPU that had been obtained from this process was characterized. Based on this, the effects of the treatment processes on the particle structure and surface composition could be determined. Optical microscopy was used to verify the size of the zinc stearate particles used in the milling of the TPU adhesive. Figure 4a shows light microscopy images of zinc stearate powder, and particles with sizes of around $25 \mu \mathrm{m}$ can be distinguished. This is in accordance with the information from the supplier that reported a particle size smaller than $74 \mu \mathrm{m}$.

\section{TPU particle size investigation}

An optical microscope was used to verify the particle size of the TPU adhesive powder and the evaluation of the results obtained is shown in Fig. 4b. Predominantly, particles with a size of approximately $250 \mu \mathrm{m}$ were identified.

The investigation using the Beckman Coulter obtained a particle size distribution with a larger amount of particles, whereby $50 \%$ of the powder volume was between 170 and $300 \mu \mathrm{m}$ for the TPU adhesive. This is in accordance with the results of the optical

Table 1 Steps in the hot melt bonding test

\begin{tabular}{ll}
\hline Steps & Procedures \\
\hline 1 & Cleaning the material (PVC) with ketone \\
2 & Primer application \\
3 & Powder adhesive (TPU) application \\
4 & Melting process by heating to $70-75^{\circ} \mathrm{C}$ \\
5 & Pressing force of 50 lb for $12 \mathrm{~s}$ \\
6 & Rest for $72 \mathrm{~h}$ \\
7 & $180^{\circ}$ angle (peel resistance test) \\
8 & $100 \mathrm{~mm}$ per min (peel resistance test) \\
9 & Three samples (used in peel test investigation) \\
\hline${ }^{*}$ Environmental conditions: $23 \pm{ }^{\circ} \mathrm{C}, 50 \%$ of humidity &
\end{tabular}


microscopy investigation. It is concluded that the solvent used for the Beckman Coulter characterization does not compromise the measured particle size. Figure 5 shows the results of the volume fractions for the untreated TPU powder obtained from a Beckman Coulter test.

Figure 6 presents an SEM image indicating the TPU particle size. According to the findings of the SEM investigations, particle sizes smaller than $500 \mu \mathrm{m}$ were predominant, which confirms the results obtained in the Beckman Coulter test and optical microscopy investigations. Moreover, non-uniform TPU particle shapes were revealed. The non-uniform particle size was formed during the milling processes due to plastic properties of the TPU polymer.

\section{EDX and XPS investigations}

An EDX investigation was applied to the TPU samples prior to the powder treatment and following the white spirit washing or plasma treatments in order to verify the differences in the surface composition. Figure 7a, b detail the percentage of zinc (a) and oxygen (b) detected while investigating distinct TPU powder adhesive samples before and after white spirit and plasma treatments.

In the evaluation of the EDX results (Fig. 7) measured with an acceleration voltage of $15 \mathrm{kV}$, the treated TPU samples showed a lower concentration of zinc compared to the original TPU powder. On the other hand, the EDX measurement performed with an acceleration voltage of $5 \mathrm{kV}$ revealed a similar zinc concentration for both the treated

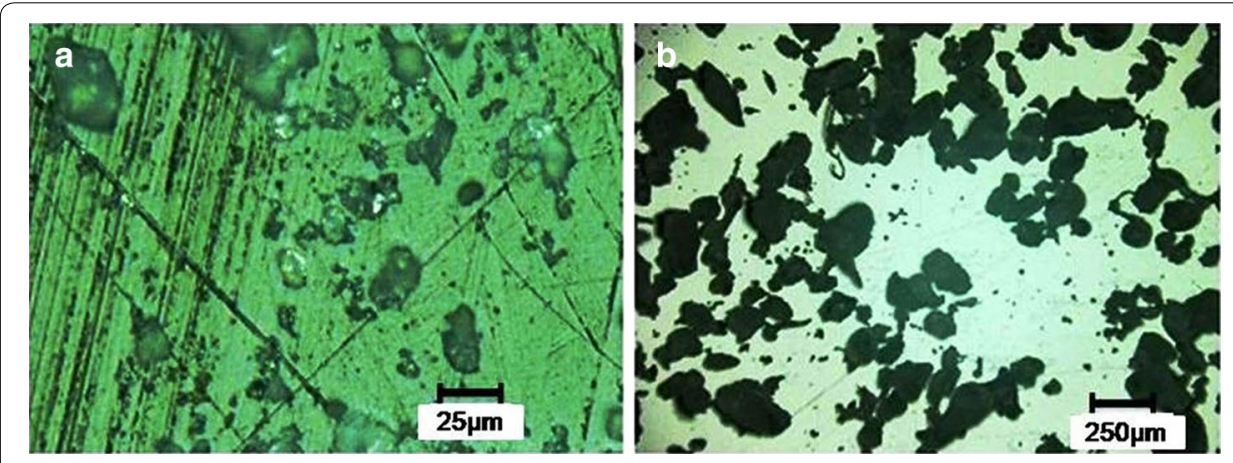

Fig. 4 Optical microscope images of zinc stearate $(1000 \times)(\mathbf{a})$ and TPU particles $(100 \times)(\mathbf{b})$

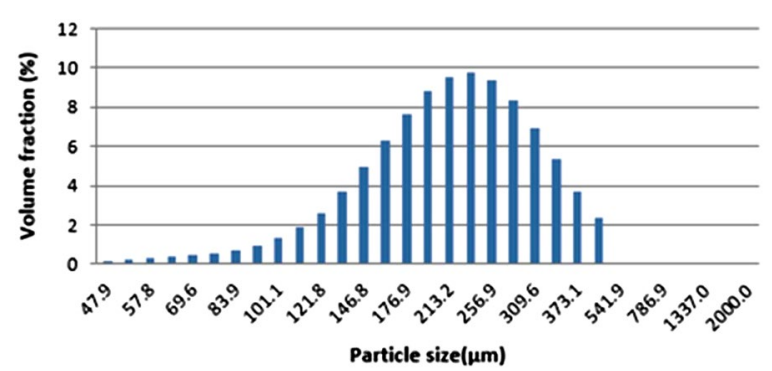

Fig. 5 Results of the Beckman Coulter test showing particle size distribution for the untreated powder TPU adhesive 
TPU powders and the original powder. Moreover, for all the TPU powders investigated the zinc concentration obtained from the measurement with $15 \mathrm{kV}$ was lower than that obtained with $5 \mathrm{kV}$. As the information depth of the measurement with $15 \mathrm{kV}$ is at least twice as big as that of the measurement with $5 \mathrm{kV}$, which is approximately $1 \mu \mathrm{m}$, the latter investigation is more surface sensitive. Therefore, these findings reveal that the zinc containing species are concentrated at the surface of the TPU particles, within a region that has a thickness of approximately $1 \mu \mathrm{m}$. Moreover, following the powder treatments the composition of the zinc containing layer appeared to differ from the state of the original powder. Possible effects of this finding are subsequently assessed by measuring the peel resistance and blocking properties to verify the good storage properties of TPU.

In principle, the plasma technique is used to activate the surface layer in different materials, including biomaterial surfaces and polymers, as an approach to improve bonding results in different substrates [20]. This is a new technique in the field of adhesives, applied to modify the zinc stearate layer around the adhesive particles. In this procedure the surface active plasma treatment may oxidize the uppermost region of

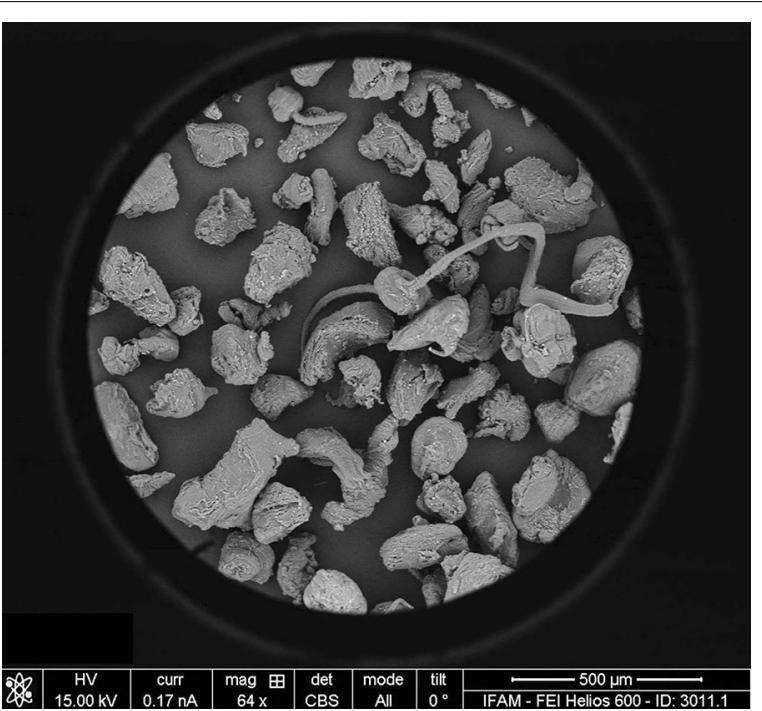

Fig. 6 SEM image showing the particle size for the powder TPU adhesive $(64 \times)$
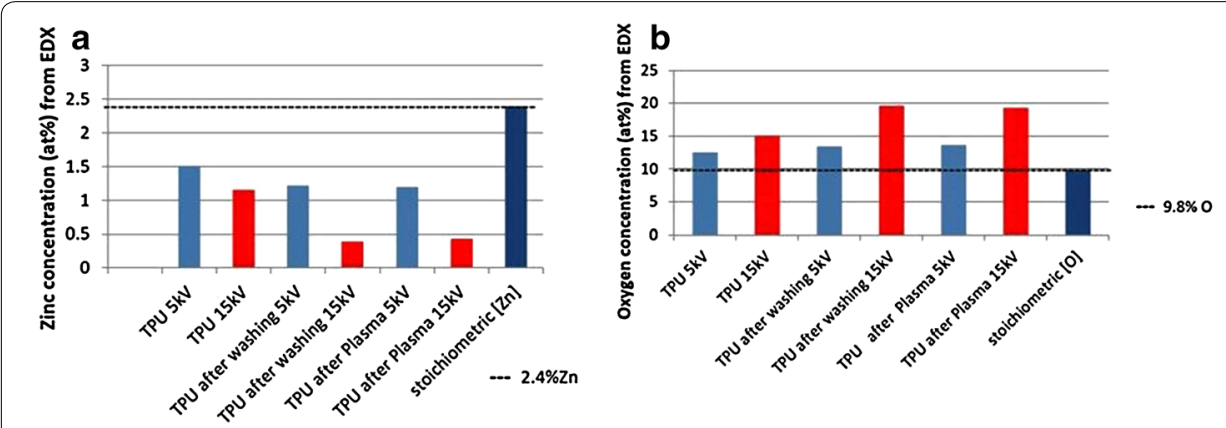

Fig. 7 Results for EDX analysis showing the atomic concentration of zinc (a) and oxygen (b) in the powder TPU adhesive before and after white spirit and plasma treatments 
the powder particles, i.e., the zinc stearate layer around the TPU adhesive. On the other hand, the washing process based on white spirit may dissolve the topmost part of the zinc stearate layer.

In Table 2 and Fig. 8 the results of XPS investigations are presented. These reveal the composition of the outermost $0.01 \mu \mathrm{m}$ layer of the TPU particles. Table 2 shows the elemental composition as an atomic concentration (given in at \%), based on XPS investigations with an information depth of $0.01 \mu \mathrm{m}$. These findings indicate that after both the white spirit washing and the plasma treatment the average surface concentration of oxygen-containing species $(\mathrm{O})$ [and the concentration ratio $(\mathrm{O}) /(\mathrm{C})$ ] as well as the zinc concentration $(\mathrm{Zn})$ is similar to that of the received state of the TPU adhesive.

$\mathrm{X}$-ray photoelectron spectroscopy investigations did not identify any significant changes in the overall atomic percentage of carbon species after treatments. However, in order to reveal the contribution of distinct carbonaceous species to the uppermost powder particle region, C1s detail XPS spectra were recorded and are shown in Fig. 8. This XPS investigation was applied for the adhesive samples before and after treatments. The C1s signals of the XPS spectra were fitted using four contributing centrosymmetric Gaussian-Lorentzian peaks around $285.0 \mathrm{eV}$ (attributed to aliphatic carbon [21]), around $285.8 \mathrm{eV}$ (attributed to carbon atoms located close to the carboxylic group [22]), around $286.8 \mathrm{eV}$ (attributed to $\mathrm{C}^{*}-\mathrm{O}$ atoms [21]), and around $289.3 \mathrm{eV}$ (attributed to carboxylic carbon [22]). The findings are in good agreement with the results reported by

Table 2 Elemental composition (atomic percentage) of the powder surfaces investigated by XPS

\begin{tabular}{llll}
\hline Sample & C (at \%) & O (at \%) & Zn (at \%) \\
\hline TPU & 89.2 & 8.7 & 1.9 \\
TPU white spirit & 90.8 & 7.6 & 1.7 \\
TPU plasma & 89.8 & 8.6 & 1.6 \\
Zinc stearate & 91.2 & 7.0 & 1.9 \\
\hline
\end{tabular}

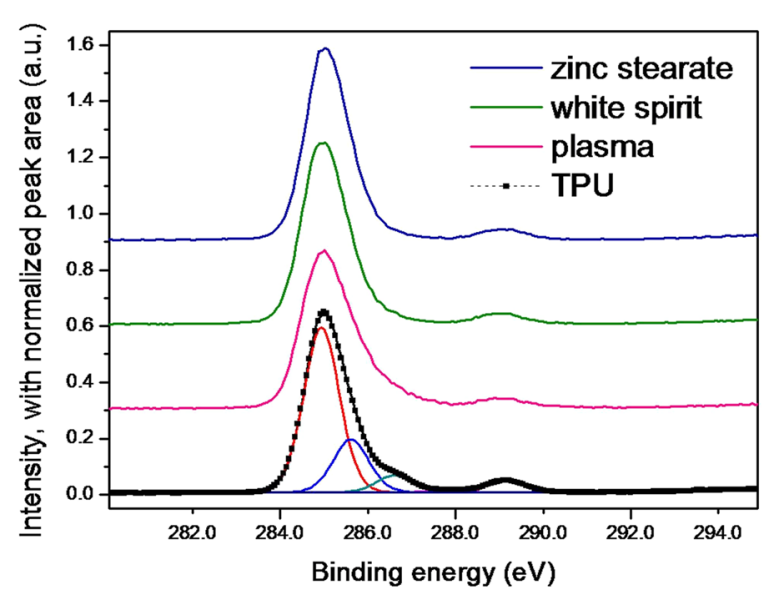

Fig. 8 C1s XPS spectra of the TPU in powder form (black line representing the measured data, red and light blue lines detailing results of a peak fitting), TPU after plasma treatment (pink line), TPU after washing with white spirit (green line) and zinc stearate (blue line) 
Gabka et al. [22], who investigated stearic acid-capped $\mathrm{Cu}-\mathrm{In}-\mathrm{Zn}-\mathrm{S}$ nanocrystals, and also indicate the presence of stearate species. Regarding zinc stearate, a composition of $\mathrm{C}_{36} \mathrm{H}_{70} \mathrm{O}_{4} \mathrm{Zn}$ may be expected, and the 1.9 at.\% of $\mathrm{Zn}$ revealed by XPS (which detects $\mathrm{C}$, $\mathrm{O}$, and $\mathrm{Zn}$ ) is a slightly lower concentration than the 2.4 at.\% expected by stoichiometric means. Therefore, the surface of the zinc stearate powder may show additional species, e.g., stearic acid.

For all TPU samples investigated a similar composition was found as for the surface of a zinc stearate powder. Consequently, following the treatments there was still a thin zinc stearate layer around the TPU powder particles.

\section{TPU thermal behavior and crystallinity}

The DSC curves obtained during the heating or cooling of the TPU powder samples enable the identification of an endothermic phase (heat absorption) or exothermic processes (heat release) [23]. The following shows the DSC curves for the samples, i.e., TPU before and after the washing treatment with white spirit and before and after the plasma treatment. Figure 9a shows the endothermal DSC curves for pure powder and after applying distinct treatments, and Fig. 9b shows the crystallization DSC curves for the TPU samples in study.

According to the melting temperature and crystallization temperature tests, which were developed using the technique previously described in the methodology, it was not possible to identify a significant difference in the TPU materials before and after washing with the white spirit solvent or after applying the plasma treatment. However, there were clear differences in the values related to the enthalpy of melting, used for verifying the crystalline fractions. The values for the enthalpies of melting, crystallization temperatures and melting temperatures are shown in the Table 3.

Comparing the melting values before and after treatments, it becomes evident that there is no phase separation using the atmospheric plasma treatment or white spirit washing, when used to modify the zinc stearate in the TPU powder.

In thermoplastic elastomers designed for use in adhesives, the crystalline fraction of polymers is also formed by soft chain segments. This means that the long chain diol segments are organized in such a way that they develop crystalline characteristics. Only polyurethanes formed by polycaprolactones and polyesters may exhibit these crystalline characteristics, due to the properties of low crystallinity of the hard segments [3]. This
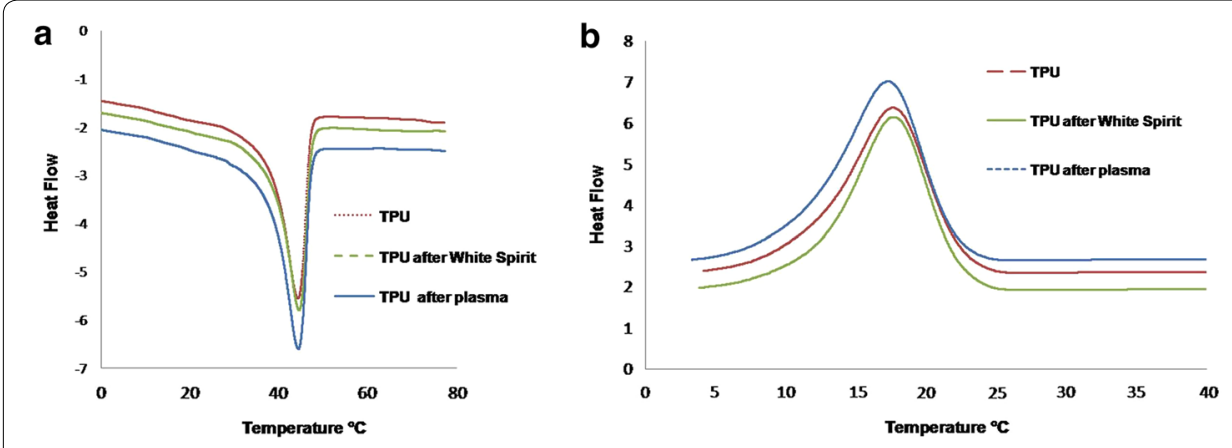

Fig. 9 DSC endothermic (a) and exothermic (b) curves for the powder TPU adhesive before and after treatment 
feature is responsible for the performance of the polymer as a thermoplastic adhesive. This work identified an increase in the percentage of crystalline fraction in the powder TPU adhesive after washing with white spirit and after the plasma treatment. The results of the crystalline fractions are shown in the Fig. 10.

\section{TPU peel resistance as a function of the treatments}

When applying the ANOVA method to the three distinct TPU powder adhesive materials (without pretreatment, after washing or after plasma treatment), a significant difference between the bonding properties of the materials is revealed. It is then possible to use the means of the bonding tests results on the Post Hoc test as Fisher's LSD type. This indicates that there is a significant difference between the bonding values in the milled TPU without treatment and those for the samples that were washed with white spirit or treated with APP. On the other hand, a comparison of the bonding results after washing with white spirit or applying plasma showed no significant differences between them. In Fig. 11, it is possible to see the differences between the results of the peel resistance, revealing that, in the case of the original TPU powder, the investigated joints had a peel resistance of $3.0( \pm 0.22) \mathrm{N} / \mathrm{mm}$. In contrast, the powder treatment by washing and the plasma treatment facilitated significantly higher values for the peel resistance, at 3.71 $( \pm 0.19)$, and $4.20( \pm 0.12)$, respectively.

These results were compared with the reference values for bonding shoes, as indicated by the Pirmasens Institute from Germany, and the bonding values increased from "Low demand bonding" to "moderate demand bonding" both after washing with white spirit and after plasma treatment [24]. In Table 4 the standard values for the categorisation of peeling resistance results may be discerned.

Table 3 Melting $\left(T_{m}\right)$ and crystallization $\left(T_{c}\right)$ temperatures, melting $\left(\Delta H_{m}\right)$ and crystallization $\left(\Delta \mathbf{H}_{c}\right)$ heat of the powder elastomers (DSC)

\begin{tabular}{lllll}
\hline Sample name & $\mathbf{T}_{\mathbf{m}}\left({ }^{\circ} \mathbf{C}\right)$ & $\mathbf{T}_{\mathbf{c}}\left({ }^{\circ} \mathbf{C}\right)$ & $\boldsymbol{\Delta H}_{\mathbf{m}}(\mathbf{J} / \mathbf{g})$ & $\mathbf{\Delta H}_{\mathbf{c}}(\mathbf{J} / \mathbf{g})$ \\
\hline TPU & 44 & 18 & 31.0 & 32.1 \\
TPU after white spirit washing & 45 & 18 & 33.6 & 33.6 \\
TPU after plasma treatment & 44 & 17 & 35.5 & 35.5 \\
\hline
\end{tabular}

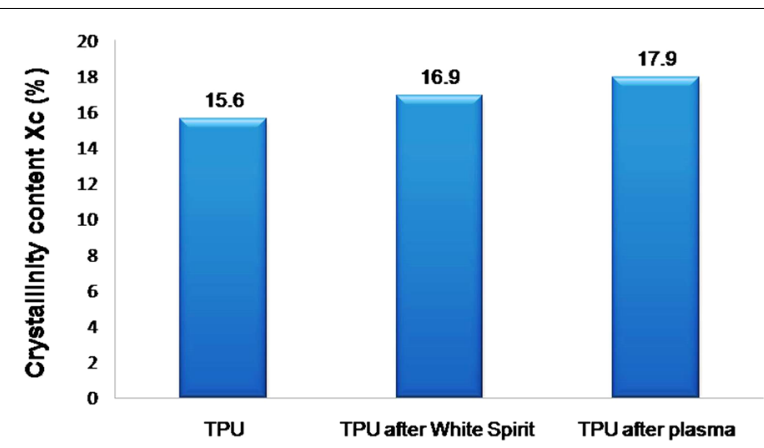

Fig. 10 Crystallinity content $\left(X_{C}\right)$ for the powder TPU adhesive before and after treatment 
In the shoe manufacturing industry, adhesive properties are crucial to ensure the manufacturing quality of the shoe. Paiva [4] described the importance of analyzing the peeling resistance of an adhesive joint subjected to various types of surface treatments using a conventional solvent-based adhesive. The present work shows an improvement in peel resistance using a TPU elastomer in powder form, replacing solvent based adhesives and obtaining a more environmentally-friendly adhesive.

Finally, the storage properties of the TPU powder material following the plasma treatment and white spirit washing were investigated by compressing a sample quantity under a special glass plate. No blocking was observed during the evaluation period, which lasted 15 days.

The treatment of TPU powder samples using the washing and plasma processes were shown to impact the crystallinity of the TPU particles and the composition of the zinc

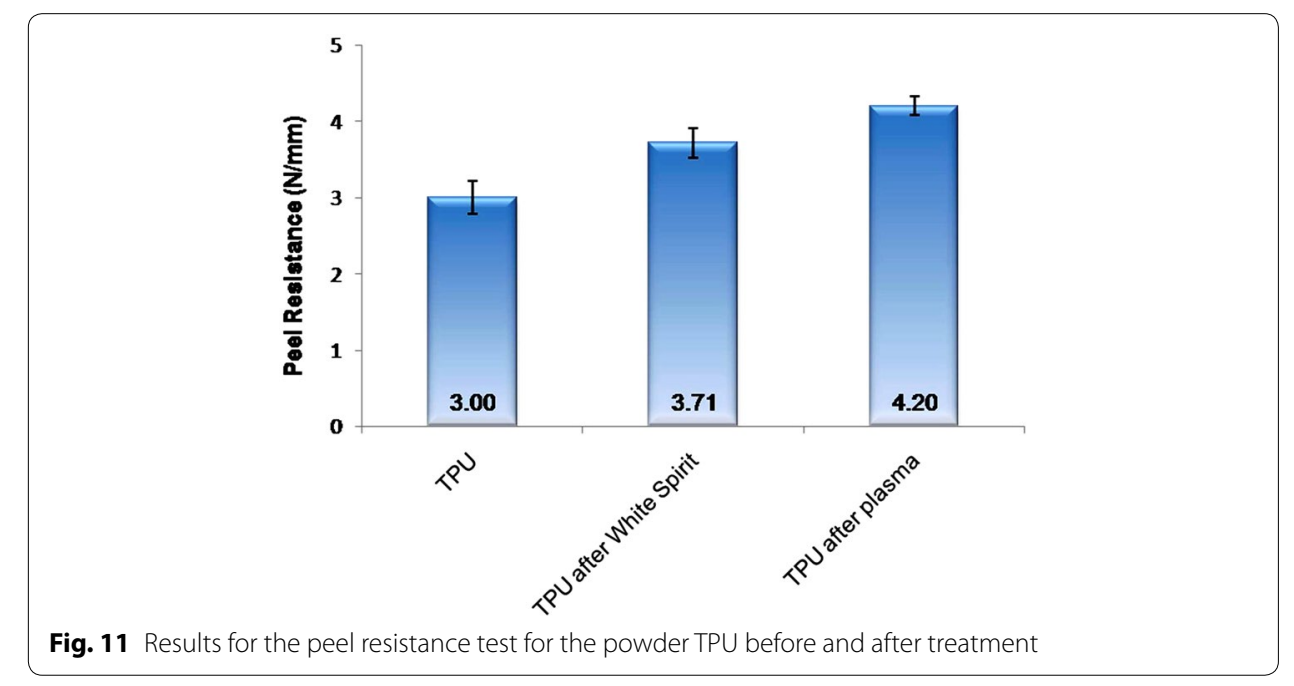

Table 4 Standard value of peeling resistance according to the shoe category

\begin{tabular}{lll}
\hline Request type & Shoe type & $\begin{array}{c}\text { Minimum peeling } \\
\text { resistance (N/mm wide) }\end{array}$ \\
\hline Shoes high demand & Children's & 6.0 \\
& Military & \\
& Security & \\
& Sports \\
& Long-distance walking & \\
Shoes average demand & Daily shoes & \\
& Walking & 4.5 \\
Shoes moderate demand & Sporting shoes & \\
& Women's social & 3.5 \\
Shoes low demand & High heels & \\
& High fashion & 2.5 \\
& Fashion footwear & \\
& Homemade & \\
& Baby's & \\
& Sandals & \\
\hline
\end{tabular}


stearate layer surrounding the TPU particles. These properties resulted from the milling process. The changes achieved by the powder treatments resulted in a significant increase in the peeling resistance of joints which consist of adhesive layers obtained from the TPU particles.

There are several polymers that are usually applied in the shoe industry and which require adherent surface treatments to achieve a satisfactory level of peel resistance; the most commonly proposed surface treatments for rubber materials include mechanical/ physical and chemical modification. These types of treatments are necessary to avoid the progressive migration of compounds from the bulk to the surface, which can occur over time [25]. Pastor et al. [25] obtained an adequate performance of adhesive joints using oxygen plasma treatment on a rubber surface. In that case the admixture of wax and zinc stearate was located inside the rubber, and there was a migration towards the surface that was especially pronounced during the bonding moment. The changes in the rubber surface were indicated by the variation in peel strength and XPS data.

In the study presented here, however, the surface of the adhesive was modified to improve the bonding performance using the similar technique of APP treatment. The findings support the suggestion of the structural model presented in Fig. 12a, b. During the milling process, TPU pellets or particles and the much smaller zinc stearate particles are mechanically deformed as a consequence of the energy impact. Following the intense mechanical contact, zinc stearate finally surrounds the TPU particles, forming a film around the adhesive polymer, as illustrated in Fig. 12a. This layer of zinc stearate formed around the particles of the TPU is in accordance with the EDX and XPS analyses, and was found to exhibit a thickness of approximately $1 \mu \mathrm{m}$. Regarding the effects of the surface treatments on the particles, Fig. 12b suggests the reduction of the zinc stearate layer thickness, and a partial removal of small zinc stearate particles. In the case of the TPU material investigated, the treatment effects are satisfactory, because the treatments modify the powder material sufficiently in order to improve peel resistance and maintain a non-blocking behavior of the powder particles.

Romero-Sanchez and Martin-Martinez [26] suggested thoroughly removing the zinc stearate at the bonding place by applying a plasma treatment to a rubber surface. In this case the peel resistance had been compromised in the presence of zinc stearate.

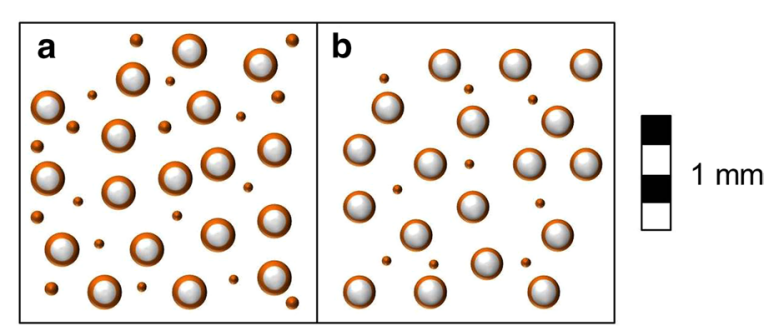
- Zinc stearate
O TPU with zinc stearate microlayer
TPU with thinner zinc stearate layer

Fig. 12 Structural model of the TPU particles in contact with zinc stearate $\mathbf{a}$ after the milling process and before treatment and $\mathbf{b}$ after washing with white spirit solvent and plasma treatment 
In a study investigating a poly- $\varepsilon$-caprolactone-based adhesive, better values of tensile strength and elongation were observed for materials with higher percentages of crystalline material [27]. TPUs are both thermoplastic and highly elastic rubbers. They consist of, essentially, linear primary polymer chains. The structure of these primary chains comprises a preponderance of relatively long and soft segments, which have been joined end-to-end by hard chain segments through covalent chemical bonds [28]. A TPU molecular chain is illustrated in Fig. 1b.

Some authors, such as R. B. Silva [29], have described studies assessing polyethylene sealing properties in dependence of the degree of crystallinity. They obtained better sealing results for materials with a lower crystalline fraction, as improving the amorphous fraction for sealing properties is favorable. In the present study the crystalline fraction was improved, and thus there was also an improvement of the hard chain segments, responsible for the best cohesion characteristic of the adhesive, and, therefore, an increase in peel resistance values could be observed.

\section{Conclusions}

This research used effective techniques to identify the differences in the zinc stearate layer before and after a white spirit wash, as a conventional treatment, and an APP treatment, as an innovative technique, as applied to a TPU powder adhesive. Using an EDX investigation, it was possible to verify a reduction of the zinc species in the layer around the TPU particles, without compromising the blocking characteristics of the TPU adhesive following treatment, as the treatments essentially only affected the zinc stearate layer. Particle size investigations with optical microscopy, Beckman Coulter and SEM indicated that the zinc stearate layer is small in comparison to the TPU particle size. Therefore, the treatments acted only on the layer containing the zinc stearate lubricant. The crystallinity test showed a higher value for the TPU adhesive powder after both the white spirit wash and plasma treatments, which was attributed to an increase in the hard segments, which are responsible for the best cohesion and, thus, better peel resistance values.

Summarizing, the present paper has illustrated that it is feasible to improve peel resistance for joints with a micronized powder adhesive containing a zinc stearate layer as a lubricant, using an innovative technique, such APP treatment, to modify the adhesive particle surface.

\section{Abbreviations \\ $\mathrm{X}_{c}$ : crystalline; $\Delta \mathrm{H}_{\mathrm{m}}$ : melting enthalpy; $\Delta \mathrm{H}_{0}$ : theoretical enthalpy; DSC: deferential scanning calorimetry; MEK: methyl ethyl ketone; PVC: polyvinyl chloride; TPU: thermoplastic polyurethane.}

\section{Authors' contributions}

GB contributed to the preparation of the TPU powder samples and also participated in the analysis and interpretation of the data, including drafting and revising the manuscript. MF participated in analyzing the data and drafting the manuscript. SS did the preparation for the particle size tests, including the evaluation and discussion of the microscopic and spectroscopic data, and undertook the lay-out and formatting of the article. MN participated in the defining and setting up of the experiments with TPU, performed the XPS investigations, and analyzed the data. AK customized and performed the Atmospheric Pressure Plasma treatment. WLC contributed to the planning of the conceptual approach, discussed and merged the data obtained, and drafted the manuscript. All authors read and approved the final manuscript.

Authors' information

GB and SS participated in the program Science without Borders from CAPES at Fraunhofer IFAM Bremen, where the experiments were performed. 


\section{Author details}

${ }^{1}$ Federal University of Rio Grande do Sul-UFRGS-PPG3M, Av. Bento Gonçalves, 9500, Porto Alegre, RS CEP: 91540-000, Brazil. ${ }^{2}$ Department of Chemistry, UFSC, Federal University of Santa Catarina, Campus Universitário Trindade, Florianópolis, SC 8040-900, Brazil. ${ }^{3}$ Fraunhofer Institute for Manufacturing Technology and Advanced Materials IFAM, Wiener Straße 12, 28359 Bremen, Germany.

\section{Acknowledgements}

The authors are grateful to Science without Borders (Ciência sem Fronteiras, G. Bockorny 248896/2013-0, and St. Stamboroski 88888.020610/2013-00) and to Coordination of Improvement of Higher Education Personnel (CAPES-Brazil) and to UFRGS—Federal University of Rio Grande do Sul (PPG3M); Federal University of Santa Catarina_UFSC—Florianópolis, SC-Brazil; Artecola Química S.A., Innovation Institute for Polymers Engineering SENAI. Moreover, the authors gratefully acknowledge the contributions by Lucas Taveira Caleiro when drawing the representation for zinc stearate, and they thank Dr. Karsten Thiel for performing SEM and EDX investigations.

\section{Competing interests}

The authors declare that they have no competing interests.

Received: 3 December 2015 Accepted: 26 February 2016

Published online: 18 March 2016

\section{References}

1. Company Merquinsa. What's a polyurethane. Barcelona: Montmeló; 2014.

2. Xiaoyan Gao BZ, et al. Synthesis and characterization of well-dispersed polyurethane/CaCO 3 . Colloids Surf A Physicochem. 2010. doi:10.1016/j.colsurfa.2010.08.036.

3. Lubrizol M. Polyuretane. Barcelona: Montmeló; 2013.

4. Paiva RM, Marques EA, Silva LF, Vaz MA. Importance of the surface treatment in the peeling strength of the joints for the shoes industry. Appl Adhes Sci. 2013. doi:10.1186/2196-4351-1-5.

5. Cloquell AP, De Meter EC. Experimental analysis of an adhesive surface grinding process. J Manuf Process. 2015. doi:10.1016/j.jmapro.2015.04.003.

6. Silva SA. Estudo do comportamento reológico dos adesivos hot melt PSA e sua relação com a composição e as propriedades adesivas. Porto Alegre: Curso de Mestrado em Engenharia, Departamento de Engenharia, UFRGS; 2008. [Master Thesis]

7. Abbott SG, Brewis DM, Manley NE, Mathieson I, Oliver NE. Solvent-free bonding of shoe-soling materials. Int J Adhes Adhes. 2003;23:325-30. doi:10.1016/S0143-7496(03)00025-3.

8. Costa HM, Visconte LL, Nunes RC, Furtado CR. Aspectos históricos da vulcanização. Polímeros. 2003;13:125-9.

9. Davanço T, Tanada-Palmu P, Grosso C. Filmes compostos de gelatina, triacetina, ácido esteárico ou capróico: efeito do pH e da adição de surfactantes sobre a funcionalidade dos filmes. Ciênc Tecnol Aliment Campinas. 2007:408-16. doi: 10.1590/S0101-20612007000200034.

10. Gujel AA. Obtenção e caracterização de artefatos elastoméricos contendo aditivos provenientes de fontes renováveis. Caxias do Sul: Universidade de Caxias do Sul; 2012. [Master Thesis]

11. Lima LM. Estudo da degradação térmica oxidativa de graxas lubrificantes. João Pessoa: Universidade Federal da Paraíba; 2009. [PhD Thesis]

12. Mattos ED, Diniz MF, Dutra RD. Técnicas FT-IR (PAS, UATR e Objetiva ATR) Aplicadas à Caracterização de EPDM Modificada com Plasma. Polímeros. 2014;24:411-6. doi:10.4322/polimeros.2014.015.

13. Pereira da Cruz, Jorge Afonso. J Pereira da Cruz SA Mello, Jardel Luis. Footwear parts gluing process using powder adhesive. Int Cl. A43D 25/18. EP 2702889 A1. 05.03.2014 Bulletin 2014/10.

14. Hair JF, Tathan RE, C RL. Análise Multivariada de Dados, vol. 3. Porto Alegre: Bookman; 2005.

15. Montgomery DC. Design and analyses of experiments. Hoboken: Wiley; 1997.

16. ASTM D 3418-12-1. Standard test method for transition temperatures and enthalpies of fusion and crystallization of polymers by differential scanning calorimetry. [S.I.]. 2012.

17. Correa AX. Avaliação da Influencia da degradação com peróxido nas propriedaddes de polipropileno randômico com eteno ou buteno. Porto Alegre: Universidade Federal do Rio Grande do Sul; 2007. [Master Thesis]

18. Pistor V. Síntese de Poliuretano Termoplásticopor Extrusão Reativa Contendo Diferentes Teores do Oligômero Poliédrico Isooctil trisilanol_POSS. Caxias do Sul: UCS; 2010. [Master Thesis]

19. ABNT NBR 10456:2012 Adesivos para calçados e correlatos: Determinação da resistência da colagem. Brasil.

20. Lee JH, Kwon JS, Kim YH, Choi EH, Kim KM, Kim KN. The effect of enhancing the surface energy os a polyestyrene plate by air atmospheric pressure plasma jet on early on attachment of fibroblast under moving incubation. Thin Solid Films. 2013. doi:10.1016/j.tsf.2013.04.105.

21. Wei $Q$ et. al. Multivalent anchored and crosslinked hyperbranched polyglycerol monolayers as antifouling coating for titanium oxide surfaces. Colloids Surf B Biointerfaces 2014:684-92. doi:org/10.1016/j.colsurfb.2014.08.001.

22. Gabka G, et al. Ligand exchange in quaternary alloyed nanocrystals-a spectroscopic study. Phys Chem Chem Phys. 2014;16:23082-8. doi:10.1039/c4cp03850a.

23. Melo JW. Produção e caracterização de pó de PET—Poli (Tereftalato de etileno), obtido a partir de garrafas pós-consumo. Belo Horizonte: Rede Temática em Engenharia de Materiais UFOP_CETEC_UEMG; 2004. [Master Thesis]

24. Senai Calçados. Bonding values indicated by the Pirmasens Institute. Germany.

25. Pastor-Blas MM, Martines JM, Dillard JG. Surface characterization of synthetic vulcanized rubber treated with oxygen plasma. Surf Interface Anal. 1998;26:385-99. 
26. Romero-Sanchez MD, Martin-Martinez JM. Surface modifications of vulcanized SBR rubber by treatment with atmospheric pressure plasma torch. Int J Adhes Adhes. 2006. doi:10.1016/j.ijadhadh.2005.05.002.

27. Choi WY, Lee CM, Park HJ. Devlopment of biodegradable hot-melt adhesive based on poly-E-caprolactone and soy protein isolate for food packaging system. Sci Direct. 2006. doi:10.1016/j.Iwt.2005.04.012.

28. Petrie EM. Crystalline thermoplastic polyurethane adhesives. Adhesive and sealants. 2005.

29. Silva RB. Correlação Estrutura/propriedade de polietileno linear de baixa densidade: influência na temperatura de selagem. Porto Alegre: Universidade Federal do Rio Grande do Sul; 2010.

Submit your manuscript to a SpringerOpen ${ }^{\circ}$ journal and benefit from:

- Convenient online submission

- Rigorous peer review

- Immediate publication on acceptance

- Open access: articles freely available online

- High visibility within the field

- Retaining the copyright to your article

Submit your next manuscript at $\boldsymbol{\nabla}$ springeropen.com 